\title{
Quantum-fluctuation-induced collisions and subsequent excitation gap of an elastic string between walls
}

\author{
Yoshihiro Nishiyama* \\ Department of Physics, Faculty of Science, \\ Okayama University, Okayama 700-8530, Japan.
}

(Dated: November 6, 2018)

\begin{abstract}
An elastic string embedded between rigid walls is simulated by means of the density-matrix renormalization group. The string collides against the walls owing to the quantum-mechanical zero-point fluctuations. Such "quantum entropic" interaction has come under thorough theoretical investigation in the context of the stripe phase observed experimentally in doped cuprates. We found that the excitation gap opens in the form of exponential singularity $\Delta E \sim \exp \left(-A d^{\sigma}\right)(d$ : wall spacing) with the exponent $\sigma=0.6(3)$, which is substantially smaller than the meanfield value $\sigma=2$. That is, the excitation gap is much larger than that anticipated from meanfield, suggesting that the string is subjected to robust pinning potential due to the quantum collisions. This feature supports Zaanen's "order out of disorder" mechanism which would be responsible to the stabilization of the stripe phase.
\end{abstract}

PACS numbers: 74.72.-h High-Tc compounds, 05.10.-a Computational methods in statistical physics and nonlinear dynamics, 46.70.Hg Membranes, rods and strings, 05.70.Jk Critical point phenomena. 


\section{INTRODUCTION}

Recently, Zaanen brought up a problem of "quantum string," which is a linelike object subjected to line tension, and it wanders owing to quantum zero-point fluctuations1. 2 . Central concern is to estimate the interaction among strings as it wanders quantum-mechanically and undergoes entropy-reducing collisions with adjacent neighbors. Statistical mechanics of quantum-string gas would be responsible to the low-energy physics of the stripe phase observed experimentally in doped cuprates how the stripe pattern acquires stability. Actually, a good deal of theoretical analyses had predicted tendency toward stripe-pattern formation 6.6.6. However, ab initio simulations on the $t-J$ model still remain controversial about that issue $19,12,12,13,14$.

In the series of papers 1 , 2 , the authors pointed out that the "quantum entropic" interaction lies out of conventional picture, and it would rather give rise to the solidification of the gas of strings; namely, the stripe phase is stabilized by mutual collisions. (In a different context, the entropic interaction was explored in Refs. 15,16.) Their analysis1 1 . 16 based on the Helfrich approximation 17 , which has been very successful in the course of studies of stacked membranes under thermal undulations. Based on this approximation, they reveled a significance of long-wavelength fluctuations. Note that in the conventional picture, on the contrary, the string is "disordered" as in the Einstein-like view of crystal, and only short-wavelength fluctuations contribute to collisions.

To be specific, the key relation in their theory is the following,

$$
f=C \frac{B}{\Sigma d^{2}}\left(\log \left(\frac{\Sigma d}{B}\right)+C^{\prime}\right)
$$

with collision-induced energy cost $f$ (per unit volume) and elasticity modulus (with respect to the compression of string intervals) $B=d^{2} \partial^{2} f / \partial d^{2}$; see the Hamiltonian (2) as well. The logarithmic term signals the significance of long-wavelength fluctuations. The relation (1) yields remarkable consequences. For instance, the elastic modulus is given by the stretched exponential form $B \sim \exp \left(-A d^{2 / 3}\right)$ 胆. It is noteworthy that the elasticity modulus $B$ is much larger than that of meanfield $B \sim \exp \left(-A d^{2}\right)$. Hence, the result indicates that contrary to our naive expectations, short-wavelength modes are suppressed owing to collisions, and the collisions rather contribute to the solidification of the string gas; namely, the stripe phase is stabilized by the "order out of disorder" mechanism 1 , 目. 
In our preceding paper 18 , we have verified the above relation (11), and demonstrated that the elastic modulus is actually governed by the stretched exponential form $B \sim$ $\exp \left(-A d^{0.808(1)}\right)$. We have carried out the first-principle simulation by means of the densitymatrix renormalization group 1920,21: We put a quantum string between rigid walls with spacing $d$, and measured its repelling interaction. (This technique has been utilized in the course of studies of fluctuation pressure of stacked membranes22,2324.) The Hamiltonian is given by,

$$
\mathcal{H}=\sum_{i=1}^{L}\left(\frac{p_{i}^{2}}{2 m}+V\left(x_{i}\right)\right)+\sum_{i=1}^{L-1} \frac{\Sigma}{2}\left(x_{i}-x_{i+1}\right)^{2} .
$$

Here, $x_{i}$ denotes the operator of transverse displacement of a particle at $i$ th site, and $p_{i}$ is its conjugate momentum. They satisfy the canonical commutation relations $\left[x_{i}, p_{j}\right]=\mathrm{i} \hbar \delta_{i j}$, $\left[x_{i}, x_{j}\right]=0$ and $\left[p_{i}, p_{j}\right]=0 . V(x)$ is the rigid-wall potential with spacing $d$;

$$
V(x)=\left\{\begin{array}{l}
0 \quad \text { for } 0 \leq x \leq d \\
\infty \text { otherwise }
\end{array}\right.
$$

$\Sigma$ denotes the line tension which puts particles into line. Classical version of this Hamiltonian has been used as a model for line dislocations and steps on (vicinal) surfaces2, 26,27. Note that for sufficiently large $\Sigma$, one can take continuum limit, with which one arrives at fieldtheoretical version of quantum stringe8

In the present paper, we are concerned in the excitation (mass) gap due to the collisions. We postulate the following exponential singularity,

$$
\Delta E \sim \exp \left(-A d^{\sigma}\right)
$$

for sufficiently large $d$. In Ref. 2, the gap (crossover temperature) $T_{0}$ is calculated in the form $T_{0} \propto \sqrt{B}$; namely, one should obtain $\sigma=2 / 3$. This result cannot be understood in terms of the meanfield picture yielding much smaller mass gap $\sigma=2$. (We will outline this picture in the next section.) The purpose of this paper is to judge the validity of those scenarios by performing first-principle simulations. Our result is $\sigma \approx 0.6(3)$ for $\Sigma=0.5-4$.

The rest of this paper is organized as follows. In the next section, we argue physical implications of the singularity exponent $\sigma$. We introduce the viewpoint of critical phenomena in order to interpret the gap formula (田). The meanfield argument is also explicated. In Section [II, we perform numerical simulations, and show evidences of the breakdown of meanfield. In the last section, we give summary and discussions. 


\section{INTERPRETATION OF THE SINGULARITY EXPONENT $\sigma$ - A STATISTICAL-MECHANICAL OVERVIEW}

In Introduction, we have overviewed the quantum string in the context of the stripe phase. In this section, we will survey different aspects of the quantum string in terms of statistical mechanics. This viewpoint is utilized in the simulation-data analyses in the succeeding section III.

In the path-integral picture, a quantum string spans a "world sheet" as time evolves 29. Therefore, a quantum string is equivalent to the random surface under thermal undulations. The random surface is in the critical phase of Kosterlitz and Thouless. (In our model (2), however, there appears no "flat phase," because the "height" variables $\left\{x_{i}\right\}$ are continuous.) The presence of rigid walls is supposed to destroy the KT criticality. Therefore, the collisioninduced mass gap (4) reflects the universality class of the phase transition at $1 / d \rightarrow 0$. In that sense, our aim is to determine the universality class.

The phase transition has not been studied very extensively so far. The rigid-wall potential is, by nature, non-analytic, and hence, it is quite cumbersome to carry out perturbative analyses such as loop expansions. Moreover, the confinement due to walls destroys the applicability of the duality transformation, with which one could establish an elucidating equivalence between the random surface and the two-dimensional $X Y$ model.

Even in computer simulation, the verification of the gap formula (4) is rather troublesome. Note that the gap opens very slowly. That is, the correlation length is kept exceedingly large in the vicinity of the critical point. The correlation length would exceed the system sizes. Note that the conventional criticality exhibits the power law singularity,

$$
\Delta E \sim \xi^{-1} \sim(1 / d)^{\nu}
$$

which would be much easier to cope with. We will overcome the difficulty through resorting to the density-matrix renormalization group 19,20,21; with this technique, we treat system sizes up to $L=42$.

Now, we are in a position to discuss the meaning of the singularity exponent $\sigma$. The correlation length is proportional to the inverse of the excitation (mass) gap; namely, $\xi \sim$ $\exp \left(A d^{\sigma}\right) . \quad \xi$ sets the characteristic length scale of the collision intervals. Therefore, in the length scale $l=\xi$, the random surface fluctuates freely. And so, the mean fluctuation 
deviation is calculated,

$$
\sqrt{\left\langle x_{i}^{2}\right\rangle} \sim(\log l)^{1 / 2} \sim d^{\sigma / 2}
$$

From this relation, we see that $\sigma$ reflects an amount of fluctuation amplitudes. In the meantime, the meanfield picture insists $\sqrt{\left\langle x_{i}^{2}\right\rangle} \propto d$. (More precisely, an artificial mass term is included so as to enforce the condition; see Refs. 16,22.) Hence, one should obtain,

$$
\sigma=2
$$

in the meanfield picture.

On the contrary, as is mentioned in Introduction, recent theoryt 1 predicts much smaller singularity exponent $\sigma=2 / 3$, from which one obtains a counterintuitive result $\sqrt{\left\langle x_{i}^{2}\right\rangle} \sim$ $d^{1 / 3} \ll d$. The result indicates that the string is straightened macroscopically by collisions: This is quite reminiscent of the aforementioned claim that the infrared modes are still active withstanding the collisions 1 目. Similar, but slightly modest, exponent $\sigma=1$ was reported (in a different context) with analytical calculations 15.16 .

\section{NUMERICAL RESULTS AND DISCUSSIONS}

In this section, we perform first-principle simulations so as to estimate the singularity exponent $\sigma$. First, we will explicate the simulation algorithm.

\section{A. Details of the density-matrix renormalization group}

The density-matrix renormalization group has been utilized for the purpose of treating very large system sizes 19,20 . The technique is based on an elaborate reduction of the Hilbertspace bases, and it has been applied to various spin and electron systems very successfully 21 . Meanwhile, the technique had become applicable to bosonic systems such as phonons and oscillators 30,31,32,33. (Note that the full exact diagonalization does not apply, because even a single oscillator spans infinite-dimensional Fock space. The huge dimensionality overwhelms the computer memory space.) Here, we employ the density-matrix-renormalization technique in order to diagonalize the quantum string (2). Full account of technical details is

presented in our preceding paper 18 . In this paper, we will outline the simulation algorithm with an emphasis on the modification to calculate the excitation gap precisely. 
To begin with, we need to set up local (on site) Hilbert-space bases: Provided that the line tension $\Sigma$ is turned off, oscillators are independent, and each of them reduces to the text-book problem of "particle in a box." Hence, the full set of eigensystems is calculated easily. In this way, we set up the intra-site Hilbert space with use of the low-lying $M=12$ states, and discarded the others.

Secondly, provided that those intra-site bases are at hand, we are able to apply the density-matrix renormalization group. The total system consists of block, site, site and block. The left-half part (block and site) is renormalized into a new block with reduced dimensionality $m$; we set, at most, $m=50$. More precisely, those $m$ bases are chosen from the eigenvectors of the density matrix for the left-half part,

$$
\rho=\operatorname{Tr}_{\mathrm{R}}|\Psi\rangle\langle\Psi|
$$

with the largest $m$ eigenvalues. $\operatorname{Tr}_{\mathrm{R}}$ denotes the partial trace with respect to the right-half part, and $|\Psi\rangle=|0\rangle+|1\rangle$ with the ground state $|0\rangle$ and the first excitation state $|1\rangle$ of the total system. Note that in our previous simulation 18 , we simply set $|\Psi\rangle=|0\rangle$, because we were just concerned in the ground state properties.

To summarize, we had carried out truncations of bases through two steps: One is the truncation of the intra-site bases $M$, and the other is the truncation of the "block" states $m$ through the density-matrix renormalization. Each of these procedures is monitored carefully, and the relative error of low-lying energy levels is kept within $10^{-7}$; see Ref. 18 for details.

We have repeated renormalizations 20-times. The system size reaches $L=42$.

\section{B. Excitation energy gap}

We have plotted the energy gap against the inverse of wall spacing $1 / d$ for several values of line tension $\Sigma$; see Fig. 1. From the plot, we see that the gap opens extremely slowly; namely, in the range $1 / d \lesssim 0.3$, the mass gap $\Delta E$ is maintained to be very small. This is a typical signature of the exponential singularity (田) rather than the power law (5). For $1 / d<0.2$, the simulation does not continue, because the numerical diagonalization fails in resolving the nearly degenerate low-lying levels.

In the inset, we have presented the logarithmic plot. The data exhibit convex curves. Hence, we see that the singularity exponent $\sigma$ would not exceed $\sigma=1$. As is mentioned in 
Section [I], the meanfield argument predicts $\sigma=2$. Therefore, we found that the collisioninduced gap cannot be understood in terms of the meanfield picture.

\section{C. $\beta$-function analyses}

The criticality is best analyzed by the $\beta$-function. The $\beta$-function describes the flow of a certain controllable parameter (in our case, $\delta=1 / d$ ) with respect to the infinitesimal rescaling of the unit of length; namely,

$$
\beta(1 / d)=\frac{\xi(1 / d)}{\xi^{\prime}(1 / d)}=\left(\frac{\mathrm{d}}{\mathrm{d}(1 / d)} \ln \xi\right)^{-1},
$$

with correlation length $\xi$. Because the mass gap is the inverse of the correlation length, we obtain,

$$
\beta(1 / d)=-\left(\frac{\mathrm{d}}{\mathrm{d}(1 / d)} \ln \Delta E\right)^{-1},
$$

with excitation gap $\Delta E$.

As is emphasized in Section II, the gap formula (1) is deeply concerned with the criticality at $\delta=1 / d \rightarrow 0$. The $\beta$-function reflects the universality class of the phase transition. For instance, as for the exponential singularity such as Eq. (四), it behaves like,

$$
\beta(1 / d) \sim 1 /(A \sigma)(1 / d)^{\sigma+1}
$$

On the other hand, the conventional second-order transition (5) is characterized by the behavior,

$$
\beta(1 / d)=(1 / d) / \nu
$$

In this way, we can read off the critical exponents such as $\sigma$ and $\nu$.

As is presented in the previous subsection, the excitation-gap data $\Delta E$ are readily available. Remaining task is to perform the numerical derivatives of $\Delta E^{\prime}$. We adopted the "Richardson's deferred approach to the limit" algorithm in the text book 34 . In this algorithm, one takes an extrapolation after calculating various finite-difference differentiations. We monitored the relative error, and checked that the error is kept within $10^{-8}$. From these preparations, we can calculate the $\beta$-function from our simulation data.

We plotted the $\beta$-function $\beta(1 / d)$ in Fig. 2 for $\Sigma=2$. We see that there appear two regimes: As is indicated in the plot, in the region $1 / d \lesssim 0.3$, the $\beta$-function is best fitted by a 
power law $(1 / d)^{1.6}$. Hence, the universality would belong to the exponential singularity (11) with exponent $\sigma=0.6$. Note that such stretched-exponential behavior is suggested in the previous subsection. On the contrary, the $\beta$-function falls in the simple behavior $1 / \nu(1 / d)$ with the index $\nu=2$ for large $1 / d$; see Eq. (12). That result tells that for large $1 / d$, the gap formula enters the regime of power law (5). That is quite convincing, because for large $1 / d$,

$\Sigma$ becomes irrelevant and the system reduces 18 to the text-book problem of "particle in a box." Hence, the excitation gap is simply given by the power law (5) with the index $\nu=2$. To summarize, we found that for large $1 / d$, the physics changes so that we must look into the behavior in the vicinity of $1 / d=0$.

In order to estimate $\sigma$, we have presented the $\log$-log plot in Fig. 3 for various $\Sigma$. From the slopes, we obtain an estimate $\sigma=0.6(3)$. This estimate is far less than the mean-field value $\sigma=2$. It clearly supports Zaanen's claim $\sigma=2 / 3$ 回 2 that the collision-induced mass gap is governed by the stretched-exponential singularity. That is, the collision-induced mass gap is far larger than that anticipated from meanfield, and hence, the string is subjected to robust pinning potential due to quantum collisions; see Section 世1.

Meanwhile, we had also calculated the Roomany-Wyld approximant of the $\beta$-function 35 ,

$$
\beta=-\frac{1+\ln \left(\Delta E_{l} / \Delta E_{l^{\prime}}\right) / \ln \left(l / l^{\prime}\right)}{\left(\Delta E_{l}^{\prime} \Delta E_{l^{\prime}}^{\prime} / \Delta E_{l} \Delta E_{l^{\prime}}\right)^{1 / 2}},
$$

where $l$ and $l^{\prime}$ denote a pair of system sizes. This approximant, in general, exhibits smaller finite-size collections. In fact, we found that it converges rapidly to the thermodynamic limit. However, the resultant data are identical to those with the aforementioned formula (10) for sufficiently large $L$. Because we take advantage of very large system sizes owing to the density-matrix renormalization group, the approximant formula (13) is not particularly necessary.

\section{Mean-fluctuation deviation}

So far, we have extracted the singularity exponent directly from the excitation-gap data. However, as is mentioned in Section 【, the mean deviation of the string undulations contains an information of the exponent;

$$
\Delta=\sqrt{\left\langle x_{i}^{2}\right\rangle-\left\langle x_{i}\right\rangle^{2}} \sim d^{\sigma / 2}
$$


In Fig. 4 , we plotted the mean fluctuation width $\Delta$ for various $d$ and $\Sigma=0.5-4$. From the plot, we see that there appear two distinctive regimes as in Fig. 2. For small $d, \Delta$ is proportional to $d$, as is anticipated from a naive argument; see Section II]. However, for large $d, \Delta$ starts to bend down, indicating that the string undulation becomes suppressed. Hence, for large $d$, the conventional picture does not apply. From the slopes, we read off the exponent $\sigma / 2 \approx 0.5$. (This result supports our estimate $\sigma=0.6(3)$ rather than the meanfield value $\sigma=2$.) Hence, we see $\Delta / d \ll 1$ as for $d \rightarrow \infty$. As a consequence, we found fairly definitely that the string is straightened macroscopically due to the quantum collisions. This feature is reminiscent of the infrared divergences encountered in the theory by Zaanen1.

\section{SUMMARY AND DISCUSSIONS}

We have investigated the quantum entropic (collision induced) interaction of an elastic string confined between walls (2). We performed the first-principle simulation by means of the density-matrix renormalization group. Our aim is to estimate the singularity exponent $\sigma$ in the excitation-gap formula (画). The exponent contains a number of significant informations which are overviewed in Section [1].

In order to estimate $\sigma$, we utilized the $\beta$-function (10), which is readily accessible from our simulation data. From the asymptotic form of large $d$, we obtained the estimate $\sigma=0.6(3)$

for $\Sigma=0.5$-4; see Fig. 3. Our estimate supports the recent proposal明 that $\Delta E$ is described by the stretched exponential form with $\sigma=2 / 3$, whereas it might conflict with the meanfield value $\sigma=2$. It would be noteworthy that the elastic modulus also obeys the stretched exponential, as is shown in our previous study 18 . Hence, it is established fairly definitely that the excitation gap is also described by the stretched exponential singularity. Namely, the excitation gap is much larger than that anticipated from meanfield. Moreover, we found that the mean fluctuation width $\Delta$ is far less than the wall spacing $d$; namely $\Delta \sim d^{0.5}$. This again supports our conclusion rather than the meanfield; see Eq. (6).

From those observations, we are led to the conclusion that the string is confined by extremely robust effective pinning potential due to the quantum collisions; subsequently, large mass gap opens and $\Delta$ gets bounded. Those features are also quite consistent with the findings of the series of workst2, validating their "order out of disorder" mechanism 


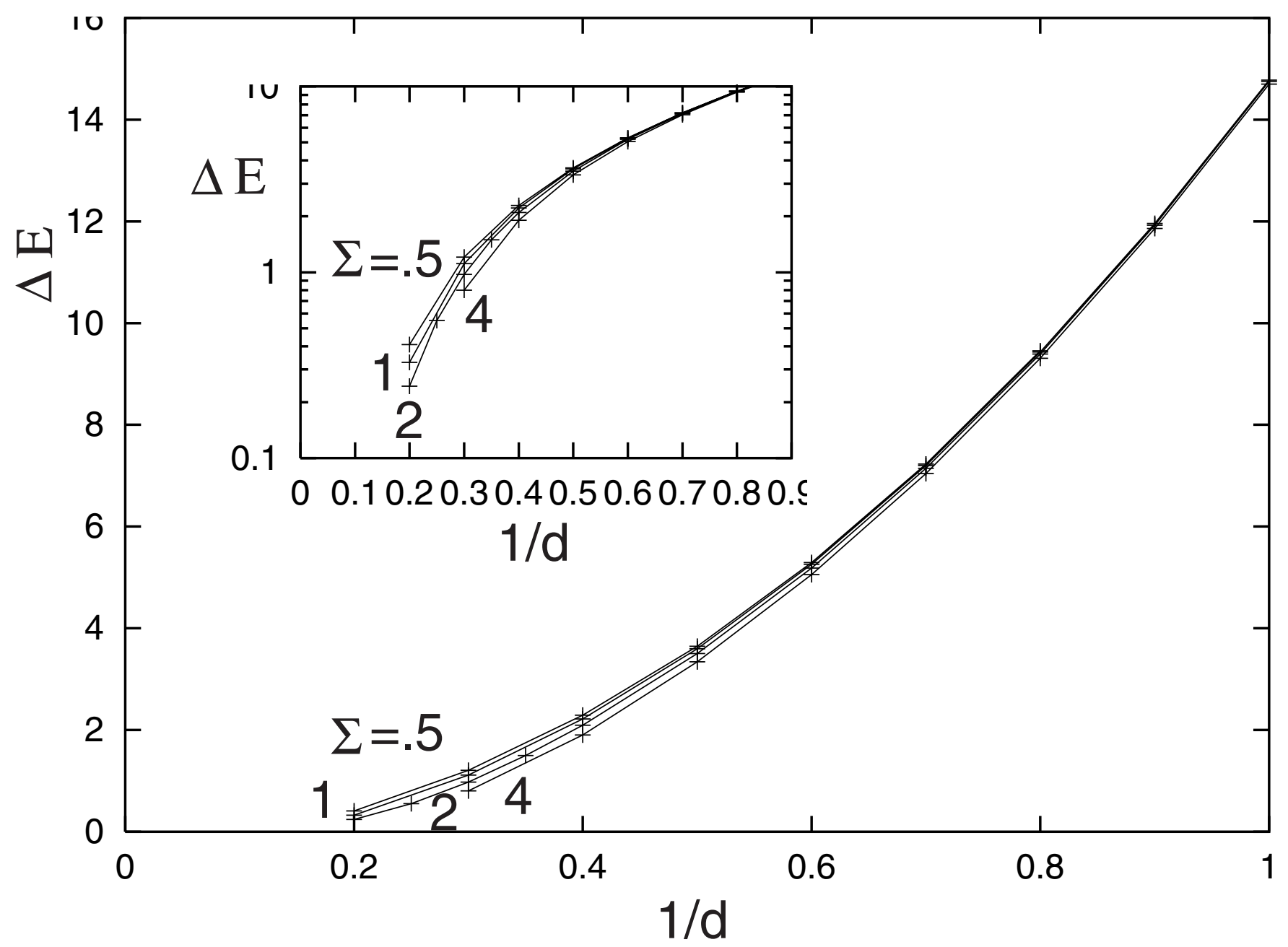

FIG. 1: Excitation gap $\Delta E$ is plotted for $1 / d$ ( $d$ : wall spacing). The excitation gap $\Delta E$ opens extremely slowly: This feature is characteristic of the universality class of exponential singularity (4) rather than the power law (5). The inset shows the logarithmic plot. The curves bend convexly. Therefore, the singularity exponent would not exceed $\sigma=1$.

responsible to the stabilization of the stripe phase.

Provided that plural strings are concerned, does the value $\sigma$ get affected? As a matter of fact, the exponent $\sigma$ is not necessarily universal, but it does exhibit continuous variation 3637 for a certain class of extended models. More specifically, in Refs. 38,39, the authors claim that for extended models with high central charges (that is, many random surfaces) exhibit various types of criticalities. Therefore, It is likely that $\sigma$ would acquire corrections for multi-string case. Extended simulation directed to this issue would be remained for future study. 


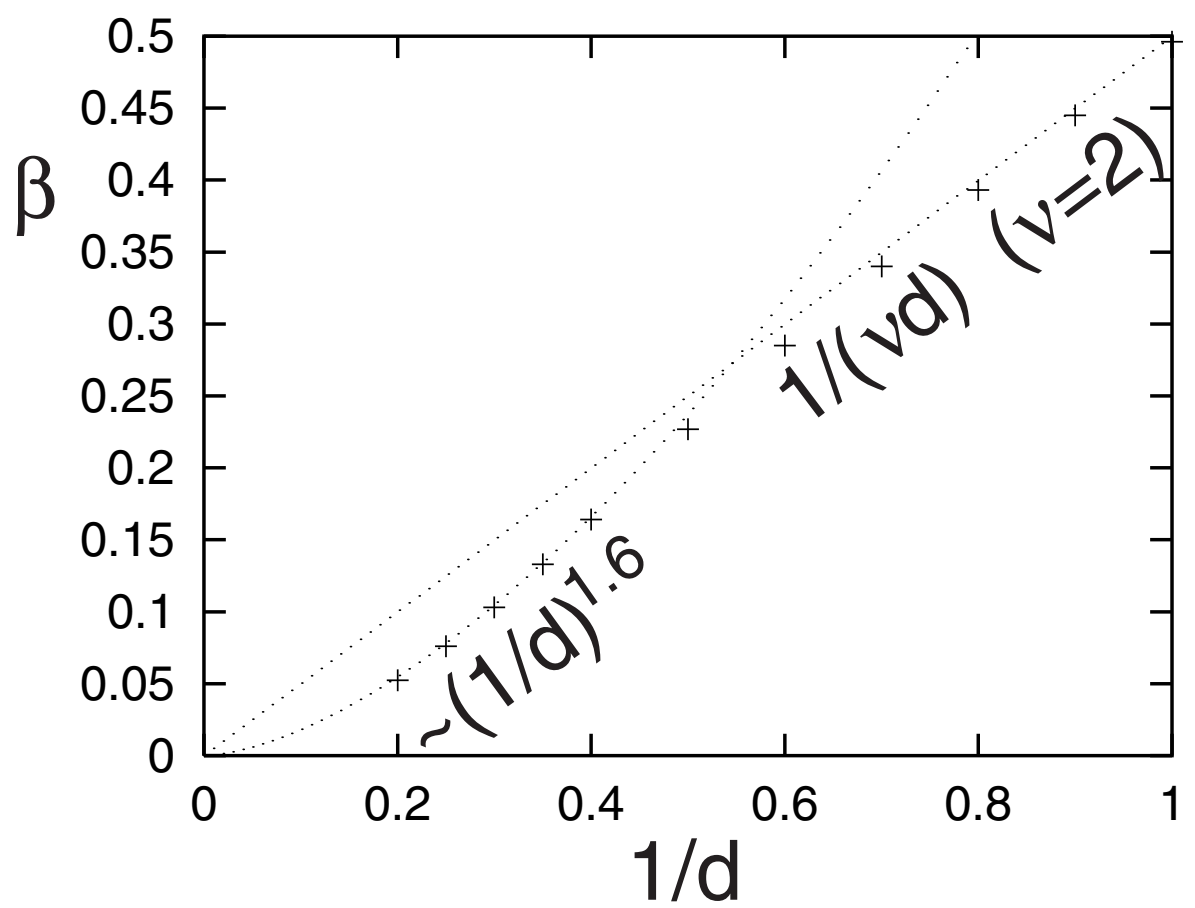

FIG. 2: $\beta$-function (10) is plotted for $\Sigma=2$. There appear two regimes with respective asymptotic forms; see text. The small-1/d behavior indicates that the transition belongs to the universality class of exponential singularity with $\sigma \approx 0.6$; see Eqs. (11) and (12).

\section{Acknowledgments}

This work is supported by Grant-in-Aid for Scientific Research Program (No. 13740240) from Monbusho, Japan. The author is grateful to Dr. T. Momoi for helpful discussions.

口

* nisiyama@psun.phys.okayama-u.ac.jp

1 J. Zaanen, Phys. Rev. Lett. 84, 753 (2000).

2 S. I. Mukhin, W. van Saarloos, and J. Zaanen, Phys. Rev. B 64, 115105 (2001).

3 J. M. Tranquada, B. J. Sternlieb, J. D. Axe, Y. Nakamura, and S. Uchida, Nature (London) 375, 561 (1995).

4 S. Wakimoto, G. Shirane, Y. Endoh, K. Hirota, S. Ueki, R. J. B. K. Yamada, M. A. Kastner, Y. S. Lee, P. M. Gehring, and S. H. Lee, Phys. Rev. B 60, R769 (1999).

5 T. Noda, H. Eisaki, and S. Uchida, Science 286, 265 (1999). 


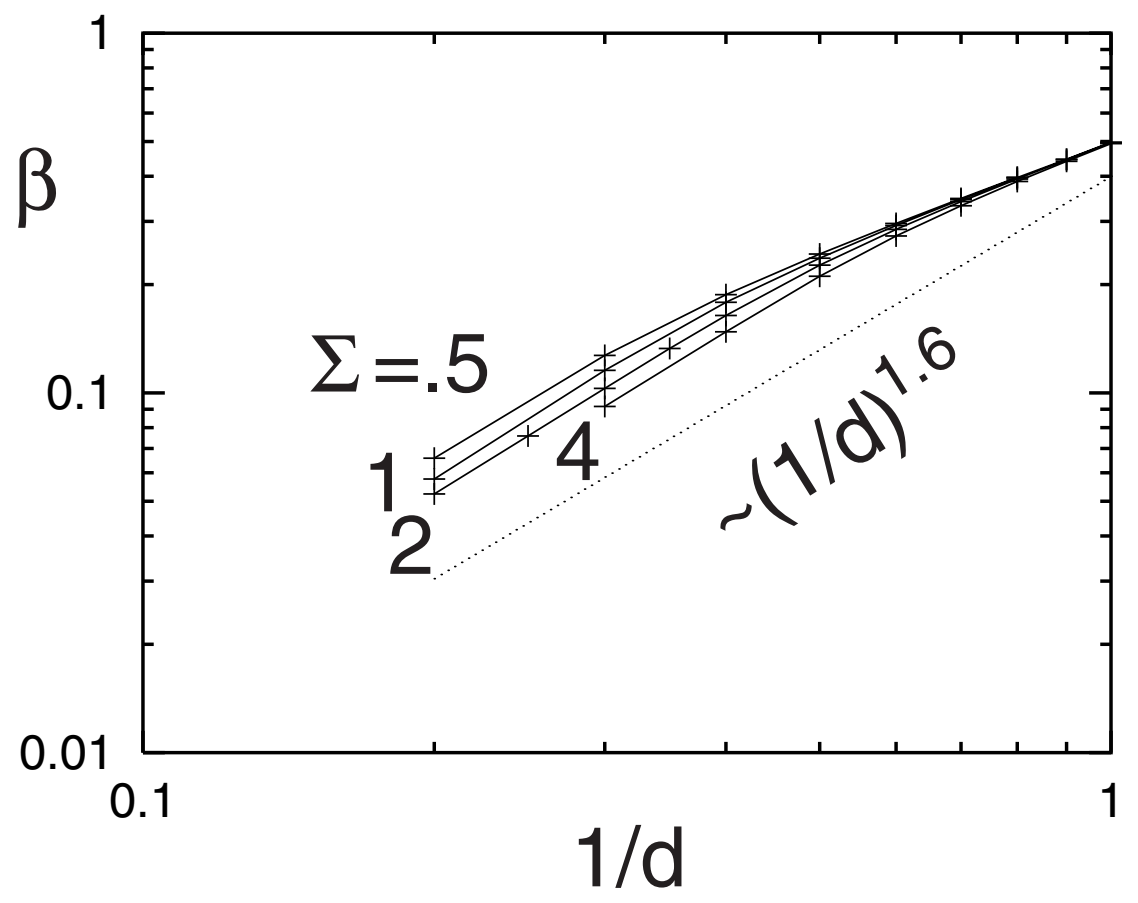

FIG. 3: Logarithmic plot of the $\beta$-function for various $\Sigma$. From the slopes, we read off the singularity exponent $\sigma=0.6(3)$; see Eq. (11). Our result indicates the breakdown of the meanfield picture $\sigma=2$.

6 J. Zaanen and O. Gunnarsson, Phys. Rev. B 40, 7391 (1989).

7 D. Poilblanc and T. M. Rice, Phys. Rev. B 39, 9749 (1989).

8 H. J. Schulz, Phys. Rev. Lett. 64, 1445 (1990).

9 M. Kato, K. Machida, H. Nakanishi, and M. Fujita, J. Phys. Soc. Jpn. 59, 1047 (1990).

10 P. Prelovsek and X. Zotos, Phys. Rev. B 47, 5984 (1993).

11 S. R. White and D. J. Scalapino, Phys. Rev. Lett. 80, 1272 (1998).

12 C. S. Hellberg and E. Manousakis, Phys. Rev. Lett. 83, 132 (1999).

13 S. R. White and D. J. Scalapino, Phys. Rev. Lett. 84, 3021 (2000).

14 C. S. Hellberg and E. Manousakis, Phys. Rev. Lett. 84, 3022 (2000).

15 J. Bricmont, A. E. Mellouki, and J. Fröhlich, J. Stat. Phys. 42, 743 (1986).

16 D. Sornette, Europhys. Lett. 2, 715 (1986).

17 W. Helfrich and R. M. Servuss, Nuovo Cimento Soc. Ital. Fis. D3, 137 (1984).

18 Y. Nishiyama, Phys. Rev. B 64, 064510 (2001).

19 S. R. White, Phys. Rev. Lett. 69, 2863 (1992). 


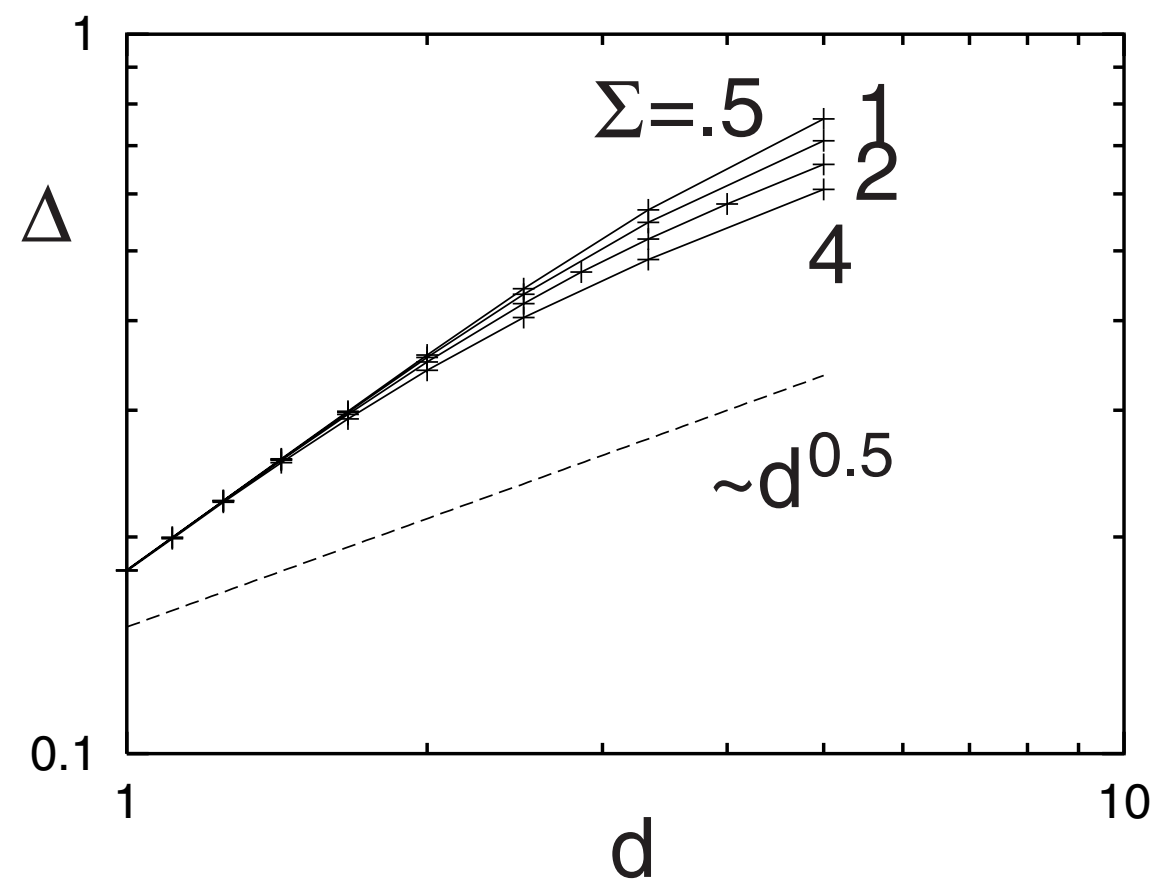

FIG. 4: Mean fluctuation width $\Delta(14)$ is plotted for wall spacing $d$. A naive argument postulates $\Delta \propto d$. However, the simulation data exhibit $\Delta \sim d^{0.5}$. We see that the string is straightened macroscopically. Our result supports the "order out of disorder" mechanism proposed by Refs. 1.,2.

20 S. R. White, Phys. Rev. B 48, 10345 (1993).

21 I. Peschel, X. Wang, M. Kaulke, and K. H. (ed.), Density matrix renormalization: a new numerical method in physics (Springer, 1999).

22 W. Janke and H. Kleinert, Phys. Lett. A 117, 353 (1986).

23 W. Janke and H. Kleinert, Phys. Rev. Lett. 58, 144 (1987).

24 S. Leibler and A. C. Maggs, Phys. Rev. Lett. 63, 406 (1989).

25 V. L. Pokrovsky and A. L. Talapov, Phys. Rev. Lett. 42, 65 (1979).

26 S. N. Coppersmith, D. S. Fisher, B. I. Halperin, P. A. Lee, and W. F. Brinkman, Phys. Rev. B 25, 349 (1982).

27 J. Zaanen, M. L. Horbach, and W. van Saarloos, Phys. Rev. B 53, 8671 (1996).

28 T. Momoi, cond-mat/0105131 (2001).

29 E. Fradkin, Phys. Rev. B 28, 5338 (1983).

30 L. G. Caron and S. Moukouri, Phys. Rev. Lett. 76, 4050 (1996). 
31 C. Zhang, E. Jeckelmann, and S. R. White, Phys. Rev. Lett. 80, 2661 (1998).

32 Y. Nishiyama, Euro. Phys. J. B 12, 547 (1999).

33 Y. Nishiyama, J. Phys. A: Math. Gen. (2002).

34 W. H. Press, S. A. Teukolsky, W. T. Vetterling, and B. P. Flannery, Numerical recipes in FORTRAN (Cambridge university press, 1992).

35 H. H. Roomany and H. W. Wyld, Phys. Rev. D 21, 3341 (1980).

36 A. P. Young, Phys. Rev. B 19, 1855 (1979).

37 D. R. Nelson and B. I. Halperin, Phys. Rev. B 19, 2457 (1979).

38 C. Itoi and S. Qin, Phys. Rev. B 63, 224423 (2001).

39 C. Itoi and H. Mukaida, Phys. Rev. E 60, 3688 (1999). 